\title{
ERRATUM
}

\section{Normal-mode-based theory of collisionless plasma waves - ERRATUM}

\author{
J. J. Ramos \\ doi:10.1017/S0022377819000400, Published by Cambridge University Press, \\ 03 July 2019
}

A recent article (Ramos 2019) contains an algebraic error that regrettably was not detected in time and invalidates some of its results. The error was made when copying the elements of the matrix $\left[\alpha_{s m}\left(v_{\|}\right)\right]_{p}^{p^{\prime}}$ given in (4.18) and (4.19), whose correct expressions should have been

$$
\begin{gathered}
{\left[\alpha_{s m}\left(v_{\|}\right)\right]_{\|}^{+}=\left[\alpha_{s m}\left(v_{\|}\right)\right]_{+}^{\|}=\frac{v_{\|} b_{s}^{1 / 2}}{\sqrt{2} v_{t h s}} \mathrm{e}^{-b_{s}}\left[I_{m-1}\left(b_{s}\right)-I_{m}\left(b_{s}\right)\right],} \\
{\left[\alpha_{s m}\left(v_{\|}\right)\right]_{\|}^{-}=\left[\alpha_{s m}\left(v_{\|}\right)\right]_{-}^{\|}=\frac{v_{\|} b_{s}^{1 / 2}}{\sqrt{2} v_{t h s}} \mathrm{e}^{-b_{s}}\left[I_{m}\left(b_{s}\right)-I_{m+1}\left(b_{s}\right)\right] .}
\end{gathered}
$$

The determinant of this matrix, given in (4.21), was calculated based on the incorrect entries. The correct result should have been

$$
\operatorname{det}\left[\alpha_{s m}\left(v_{\|}\right)\right]=0
$$

Therefore, the inverse matrix $\left[\alpha_{s m}^{-1}\left(v_{\|}\right)\right]_{p}^{p^{\prime}}$ does not exist for any $s$ or $m$ and invalidates the reported results that relied on the existence of such an inverse matrix for some $\left(s_{0}, m_{0}\right)$.

The part of the work that is invalidated is the latter part of $\S 6$, from equation (6.21) to the end of that section. This comprises the claimed proof that the complete basis of normal modes for wave propagation oblique to the background magnetic field $\left\{\Psi^{\omega, 5 \mu \rho}\right\}$ is minimal, as well as the inversion formulas for the coefficients of the expansion of any state vector in such a basis. The remainder of the work, in particular the analysis prior to equation (6.21), remains valid after correcting (4.18), (4.19) and (4.21). The results of $\S \S 7$ and 8 for wave propagation parallel and perpendicular to the background magnetic field are not affected and remain correct, because the matrix elements (4.18) and (4.19) are zero for parallel propagation and their contribution vanishes after integration over $v_{\|}$in the perpendicular propagation case. However, the completion of the normal-mode theory for electromagnetic waves propagating obliquely to the background magnetic field remains an open problem.

\section{REFERENCE}

Ramos, J. J. 2019 Normal-mode-based theory of collisionless plasma waves. J. Plasma Phys. 85, 905850401. 